\title{
Exploring Visuo-haptic Feedback Congruency in Virtual Reality
}

\author{
Benjamin Williams \\ School of Computer Science \\ University of Lincoln \\ Lincoln, $U K$ \\ bwilliams@lincoln.ac.uk
}

\author{
Alexandra E. Garton \\ School of Computer Science \\ University of Lincoln \\ Lincoln, UK \\ 14577155@students.lincoln.ac.uk
}

\author{
Christopher J. Headleand \\ School of Computer Science \\ University of Lincoln \\ Lincoln, UK \\ cheadleand@lincoln.ac.uk
}

\begin{abstract}
Visuo-haptic feedback is an important aspect of virtual reality experiences, with several previous works investigating its benefits and effects. A key aspect of this domain is congruency of crossmodal feedback and how it affects users. However, an important sub-domain which has received surprisingly little focus is visuo-haptic congruency in an interactive multisensory setting. This is especially important given that multisensory integration is crucial to player immersion in the context of virtual reality video games. In this paper, we attempt to address this lack of research. To achieve this, a total of 50 participants played a virtual reality racing game with either congruent or incongruent visuo-haptic feedback. Specifically, these users engaged in a driving simulator with physical gear shift interfaces, with one treatment group using a stick-shift gearbox, and the other using a paddle-shift setup. The virtual car they drove (A Formula Rookie race car) was only visually congruent with the stick-shift setup. A motion simulator was also used to provide synchronous vestibular cues and diversify the range of modalities in multisensory integration. The racing simulator used was Project CARS 2, one of the world's most popular commercial racing simulators. Our findings showed no significant differences between the groups in measures of user presence or in-game performance, counter to previous work regarding visuo-haptic congruency. However, the Selfevaluation of Performance PQ subscale was notably close to significance. Our results can be used to better inform games and simulation developers, especially those targeting virtual reality.
\end{abstract}

Keywords-Virtual Reality; Video Games; Visuo-haptic Feedback; Multisensory Integration; Sensory Perception;

\section{INTRODUCTION}

Virtual Reality is a rapidly advancing technology which has found utility across several disciplines, and there is an extensive body of research concerning its impact on a user's experience. The development of head-mounted displays has enabled the creation of deeply immersive user experiences as well as the sense of embodiment and presence in virtual worlds. This has been an exciting opportunity for video games development, allowing players to feel like they have been placed as agents within the world. This is in part due to the augmentation of player experiences with a level of physicality that comes from synchronising the players 'realworld' movements with those of their avatar.

Experiences in virtual reality are inherently multisensory, involving the real-time integration of sensory feedback from several modalities. For example, in virtual reality, the visual senses can be stimulated by an immersive headmounted display, synchronising the avatar's vision (the virtual camera) to the player's own head movements. Sound from headphones can also be used to concurrently provide synchronised auditory stimulation, enabling the localisation of noise sources in the virtual world. Feedback from many sensory modalities, working in harmony, can elicit a greater sense of presence and deeper sense of immersion. The more modalities which enforce this notion, the more players are immersed within the experience [1].

Our sensory systems are used to perceive the same world through different modalities. Through multisensory integration, several sensory inputs can be integrated to provide a single, holistic sensation of the world. This assumes however, a congruent relationship between the senses, working in harmony to perceive the world. This may not be the case, however, as virtual reality can often be prone to artifacts which introduce conflicting crossmodal cues. In fact, it is theorised that this disparity may cause virtual reality sickness [2]. Investigating incongruent forms of feedback is therefore crucial to understanding its impacts in virtual reality applications.

The use of virtual reality can bring about unfamiliar sensory experiences for its users. This is especially true in the case of head-mounted displays, which are often prone to hardware issues such as latency and tracking errors. It has been shown in previous work that these problems can introduce incongruent sensory information, evoking sensations of discomfort and motion sickness [1]. Investigating sensory feedback congruency and its effects is therefore a crucial component in mitigating discomfort in virtual reality settings. Whilst there have been a considerable number of works focusing on how incongruent visuo-vestibular feedback impacts virtual reality, other modalities have received comparatively little attention. For example, visuo-haptic feedback in a motion setting has largely been overlooked by researchers, despite it being a frequent component of virtual reality motion simulation. Understanding this area could better inform game developers, especially those targetting head-mounted displays and motion simulators. 


\section{BACKGROUND}

Virtual Reality (VR) is a well-established domain, with research stemming as far back as the early 1960s [3]. Since its inception, VR has been applied to several domains including medical training [4], exposure therapy [5], rehabilitation [6] and even product design [7]. Virtual reality has also found utility in video games, enabling players to be immersed in virtual environments for players to explore. VR has also been a useful tool in understanding human sensory perception and feedback, in part due to the ability to isolate specific sensory modalities. Furthermore, the prominence of virtual reality sickness has proven to be an interesting problem in this domain. As virtual reality is often a multisensory experience, research concerning human perception in VR has covered several areas, such as self-motion perception [8], somatosensory feedback [9], emotional response [10] and its effects on spatial awareness [11], to name a few. One area in particular which has received considerable attention is the use of haptic feedback (the sensation of touch) in conjunction with VR engagements.

The sensation of touch is a crucial feedback modality, and its loss can bring potentially devastating impacts on daily life. For example, the loss of the haptic senses can bring about the inability to perform tasks which rely on haptics, such as holding objects, using tools or even standing upright [12]. Haptic feedback in virtual reality typically concerns both force, and tactile feedback, and is a crucial sensory modality in immersive experiences [13]. There have been several VR haptic feedback devices developed, from pen-based haptic systems like the PHANToM OMNI [14] to pneumatic gloves enabling the sensation of resistance [15]. These haptic feedback devices have been shown to greatly improve psychomotor skill acquisition, aiding in a variety of VR training settings [16]. Haptic feedback has shown to increase user presence and task performance when compared to visual-only conditions, highlighting an important role in virtual environments [17]. Indeed, the benefits of haptic feedback are so accepted that most modern commercial VR platforms such as the Oculus Rift and HTC Vive now include vibration-based haptic feedback in their standard user interface controllers.

One interesting consideration is the congruency of sensory information between the visual and haptic senses. The integration of inputs from these two systems can aid in object recognition and perception [18]. Investigation of the visuo-haptic relationship has shown that the nervous system integrates cues similar to a maximum-likelihood estimator [19]. That is, the visual senses dominate perception when visual estimation variance is lower than haptic feedback, and vice-versa. It has also been discovered that congruent visuo-haptic inputs can aid in a more accurate perception of stimuli, over solely visual information [20]. However, some research has found that visual cues contribute more to object recognition than the haptic senses [18]. Research also suggests that the congruency of visuo-haptic feedback is also an important factor in binocular rivalry (the alternation of perception between the different images presented to each eye), reducing the strength of suppression [21]. Outside the scope of visual perception, there is also evidence that incongruent visuo-haptic feedback can negatively impact spatial matching performance [22].

Several papers have previously investigated the visuohaptic sensory relationship in the context of virtual reality. For instance, Di Luca and Mahnan conducted an investigation into the temporal perception threshold of delayed visuohaptic feedback [23]. In their experiments, participants were given a task of viewing virtual objects, which upon their touch, would receive slightly delayed vibrotactile feedback. This delay was varied, to explore the perceptable limit of temporally synchronous visuo-haptic feedback. The authors findings suggest that $50 \mathrm{~ms}$ is the perceptable limit of detecting temporal asynchrony. The dominance of visual cues in visuo-haptic object perception has also been observed in VR, specifically in regards of the appearance of the avatar's hands - with this being an important factor in how surfaces are perceived in virtual reality [18]. Congruency of visuohaptic cues, and its effects, has also been considered in virtual reality settings. This is especially important given that virtual reality is often prone to incongruent sensory cues [24], [2]. Sengul et al. specifically investigated the congruency of visuo-haptic feedback in VR, finding that congruent crossmodal cues elicited a sensation of more personal space [25]. In a similar stride, Kuschel et al. explored conflicting visual and haptic feedback, in an attempt to gauge its effects on user presence [26]. The authors find a significant effect that incongruent visuo-haptic information deteriorates the feeling of presence in users, as suggested by previous work [27]. Age also seems to be an insignificant factor in visuo-haptic integration, as observed in the work of Couth et al. [28]. In this work, the authors test conflicting and non-conflicting cues, finding no significant differences in discrimination thresholds between younger and older adults.

Whilst there have been several papers investigating visuohaptic feedback in virtual reality, there has yet to be any which consider congruency within a motion simulation platform. Furthermore, the majority of existing visuo-haptic VR experiments evaluate effects exclusively in the presence of induced visuo-haptic feedback. There is little to none which consider other forms of feedback in the integration process, such as vestibular or somatic stimuli. Given that multisensory integration is a fundamental component of presence in virtual reality [27], and that virtual reality games are inherently multisensory [2], it is surprising that visuo-haptic congruency in an interactive multisensory environment is an often overlooked topic. Exploration into the impact of visuo-haptic congruency in the presence of multisensory information is therefore warranted. This is furthered by the 
fact that virtual reality is often prone to tracking errors and latency, which could introduce visuo-haptic conflicts and discrepancies. Conducting an investigation into this topic may therefore benefit future developers of virtual reality experiences, by better understanding the impact of these conflicts in the context of multisensory settings. To examine this, we investigate visuo-haptic congruency in virtual reality, in the presence of induced vestibular feedback.

\section{EXPERIMENTAL DESIGN}

To investigate the effects of visuo-haptic congruency in a multisensory setting, a short experiment was devised and run across several days on the University of Lincoln campus. Throughout this period, a total of 50 participants were recruited to take part, of which 49 successfully finished the study (38 Male, 10 Female, 1 Other). These participants were initially pre-screened for any visual or vestibular disorders which could have heightened their susceptibility to motion sickness. Furthermore, experimental sessions were run in a public area to aid recruitment, attracting a diverse demographic of recruited participants. It is also worth noting that participants were unaware of experimental goals, and only received instruction regarding their task and how to use the apparatus.

Our experiment is similar in design to other works considering visuo-haptic congruency and its effects on presence. For example, Kuschel et al. [26] investigated this topic similarly through two groups, subjected to differing visuo-haptic conditions. Our experiment expands upon this methodology to investigate effects in a motion environment. The experiment involved participants playing a virtual reality racing game, whilst receiving vestibular cues from a motion simulator. Participants were tasked to complete two full laps of a virtual race track, with the goal of finishing the task in the shortest time possible. Using a driving simulator, peripherals similar to those of a real car could be used by the player to fully interact with the game in real-time. In particular, a USB steering wheel, pedal set and gear shifter were utilised as interaction methods with the game. An Atomic A3 2-DoF Motion Simulator was used alongside an Oculus Rift CV1 to provide real-time visual and motion feedback to players of the game. Furthermore, gear changes were facilitated through either a Fanatec ClubSport Shifter SQ V1.5, or through the paddle shifters built into the Thrustmaster TS-XW Racer steering wheel. In addition to the feedback provided by the motion simulator, the steering wheel also provided some haptic resistance. It is worth noting that motion feedback was chosen to provide a multisensory environment in the presence of visuo-haptic cues. Finally, the user controlled their acceleration via standard pedal controls mounted onto the motion platform. The user interface devices (pedals, shifter, and wheel) were as closely aligned to their virtual counterparts as feasible within the platform, with the aim of eliminating proprioceptive dispar- ity between the virtual and real world. An illustration of this setup can be found in Figure 1. The game, Project CARS 2 , was selected because it is a highly realistic simulator and has been applied to several professional training scenarios. Furthermore, it has a large selection of detailed (laserscanned) 'real-world' tracks, along with an exhaustive set of vehicles, enabling us to tailor the setup to our research objectives. Project CARS 2 is also one of the most popular commercial racing simulators, increasing our study's applicability to commercial platforms beyond laboratory setups. Furthermore, its shared memory API allows for easy access to detailed game state data. We recorded a variety of telemetry data throughout experimentation for future research and qualitative analysis. Participants played on the 'Mojave Sidewinder' track, completing two separate laps. This particular track was chosen due to its short length (to reduce overall VR exposure time) and a profile which encouraged regular gear changes (a range of straight lengths, and a variety of turn profiles). Participants also drove the 'Formula Rookie' vehicle, as it has an average difficulty rating, along with a sequential stick-shift matching the realworld setup. The Fanatec shifter was set to sequential mode, allowing us to closely align the visual information with the haptic interaction.

Throughout the experiment, two visuo-haptic conditions were explored via two interaction methods. In the first, participants changed gears via a haptic interaction method matching what was seen in the virtual world. Specifically, participants changed gear via a physical sequential stickshift (see highlight ' 1 ' in Figure 1), which was replicated by the avatar in the virtual car. In the second, a different haptic interaction method was used. In particular, a physical paddle-based gear shifter was used (two paddles situated behind the steering wheel, see highlight ' 2 ' in Figure 1), however, their avatar in the virtual car continued to use a sequential stick-shift. The difference between these two conditions allowed us to introduce an incongruity between the interaction method and visual feedback. An illustration of the in-game perspective can be seen in Figure 2.

User presence of participants was recorded following VR exposure, using the modified PQ questionnaire by Witmer and Singer [29], to understand how visuo-haptic congruency impacts measures of presence in virtual reality. Participants were initially shown how to use the test apparatus, and were informed of what to do in the study. They also were made aware that their data would be recorded throughout, but remained unaware of the nature of the experiment and our observations.

\section{REsults}

For the purpose of these results, Group A will refer to the group exposed to a visually-congruent simulation (the visual stimulus matching the physical interface) and Group B will refer to the visually-incongruent group. Lap time was used as 

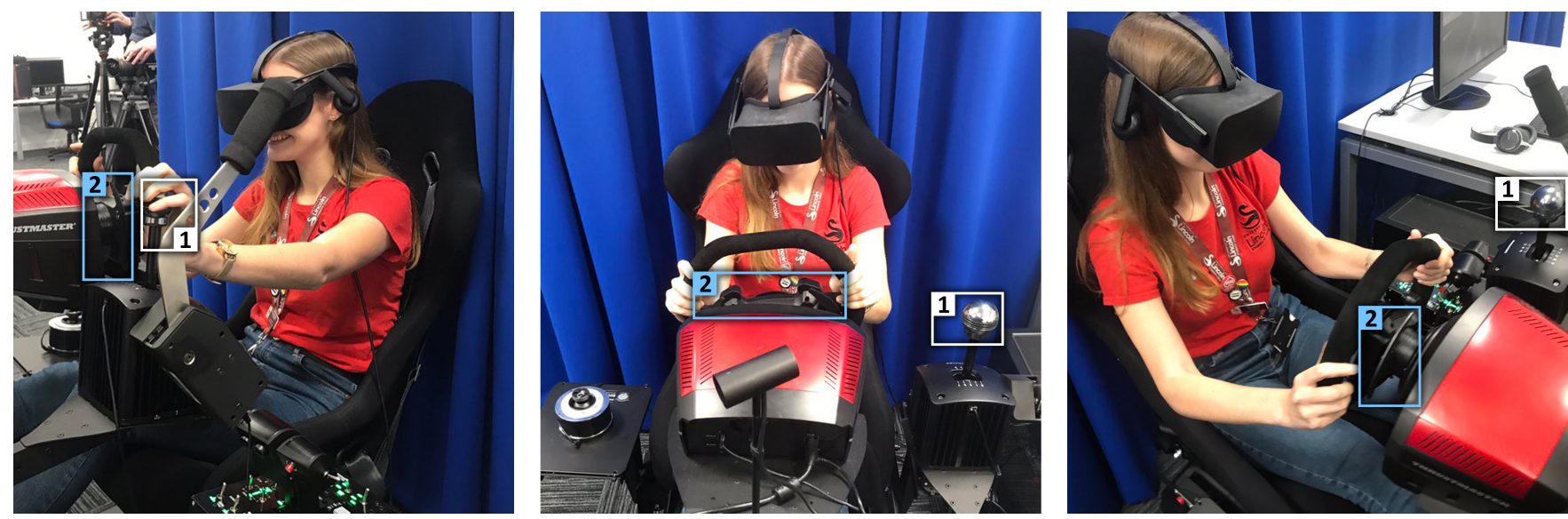

Figure 1. The experimental setup: An Atomic A3 2-DoF Motion Simulator and an Oculus Rift CV1 headset. Gear shifting is provided via either the Fanatec ClubSport stick-shift (1) or the paddle shifers built into the Thrustmaster haptic steering wheel (2).

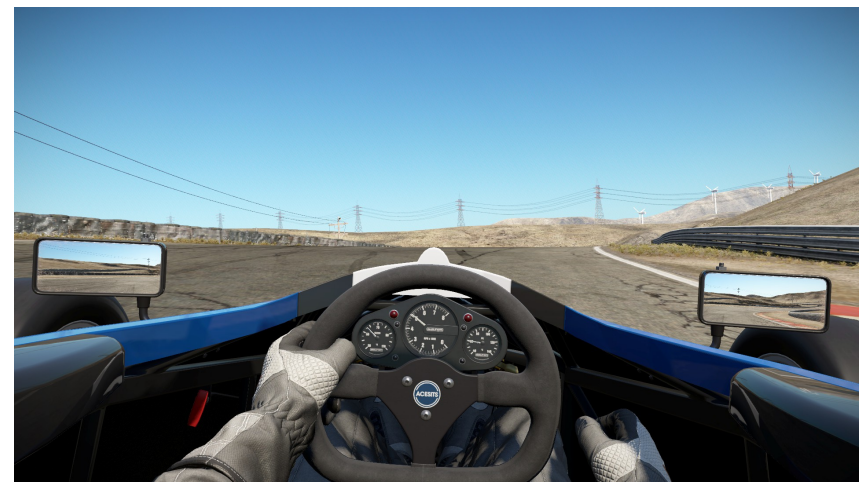

Figure 2. In-game cockpit view of the Formula Rookie, the vehicle used in our study. In this particular instance, the driver avatar is currently shifting gears with their hand on the virtual stick-shift.

a measure of player performance throughout the experiment. The first lap time of Group B $(M=127.634, S D=37.744)$ was on average, longer than Group A $(M=119.235, S D=$ 31.679 ), where $M$ denotes the mean lap time and $S D$ is the standard deviation from the mean. However, the difference between the two is not statistically significant, $t(47)=.842, p>.05$, where $t$ is the $t$ statistic of the test and 47 is the degrees of freedom. The same effect was also observed for the second lap time, with participants in Group B $(M=108.681, S D=25.834)$ performing worse than those in Group A $(M=104.558, S D=21.277)$. Again however, this difference in means was not statistically significant, $t(47)=.608, p>.05$. It is also worth noting that in high speed racing, even a fraction of a second is regarded as a considerable difference between two lap times. The average differences between Group A and B (Lap 1, 8.39 seconds; Lap 2, 4.12 seconds) would be considered a substantial overall improvement if this was the case. However, in both cases, this indicates that the visuo-haptic conditions had negligible impact on the player's performance throughout the experimental task. Furthermore, there did seem to be some improvement from the first lap to the second, likely due to practice and familiarity with the VE.

A correlation analysis of participant data further established confidence in this observed effect, showing no relationship between human factors and lap time. A full list of variables correlated can be seen in Table I. A list of significant correlations which we expected to observe can be found in Table II. Interestingly, no significant correlations were found between lap time and measures of prior experience with virtual reality, racing (either virtual or real) or motion simulators. Another relationship was found between the first and second lap times $\left(r_{s}=.456, p<.05, N=49\right)$ highlighting a reduction in lap time in the second lap, suggesting that acclimatisation and practice possibly improved times in both groups. It is worth noting that $r_{s}$ denotes the correlation coefficient, and $N$ is the sample size used in the test.

Table I

A LIST OF DEMOGRAPHIC \& PLAYER PERFORMANCE VARIABLES RECORDED THROUGHOUT THE STUDY. NOTE THAT Q* IN THIS TABLE IS A TRUNCATION OF 'QUESTIONNAIRE'.

\begin{tabular}{l|l|l} 
Factor & Type & Measured by \\
\hline \hline Subject has driving licence? & True/False & Pre-study Q* \\
Prior VR experience & Likert [1,5] & Pre-study Q* \\
Prior Motion Simulator experience & Likert [1,5] & Pre-study Q* \\
Prior Racing (virtual) experience & Likert [1,5] & Pre-study Q* \\
Prior Racing (real) experience & Likert [1,5] & Pre-study Q* \\
Prior Driving experience & Likert [1,5] & Pre-study Q* \\
Age group & Group [1, 4] & Pre-study Q* \\
Gender & Group [1, 4] & Pre-study Q* \\
Lap 1 time in seconds & Real number & Study software \\
Lap 2 time in seconds & Real number & Study software
\end{tabular}

Perhaps the most interesting results can be found when comparing measures of user presence between the two groups. Presence was measured using Witmer and Singer's PQ questionnaire [29], which records presence across several 
Table II

THE LIST OF SIGNIFICANT CORRELATIONS FOUND WHEN CORRELATING DEMOGRAPHIC \& PERFORMANCE METRICS, WHERE $r_{s}$ IS THE CORRELATION COEFFICIENT AND $p$ IS THE P-VALUE OF THE TEST. IN ALL CASES, $N=49$.

\begin{tabular}{l|l|l|l} 
Variable 1 & Variable 2 & $r_{s}$ & $p$ \\
\hline \hline Lap 1 time & Lap 2 time & .456 & .001 \\
Prior VR exp. & Prior motion sim. exp. & .457 & .001 \\
Weekly hours driving & Age group & .433 & .002 \\
Weekly hours driving & Has driving licence? & .514 & .000
\end{tabular}

subscales mapped to different aspects of the experience. No significant differences in all recorded subscales were found between the two groups $(\alpha=.05)$, meaning participants experienced similar levels of presence irrespective of the visuo-haptic condition they were subjected to. The same is also true of the overall presence score. More detailed results can be seen in Table III. Although no significance was found across each test, the subscale with a $p=.076$ closest to $\alpha$ was Self-evaluation of Performance (SEOP), with a difference of $|\alpha-p|=.026$. The SEOP subscale specifically concerns a participant's feeling of competence to perform tasks in the virtual environment (VE) [30]. Although not significant, it is not only interesting that this subscale had a substantially larger effect size to others, but that $p$ is very close to the significance level $\alpha$. It should also be noted that those interacting with the game using a haptic modality matching visual cues had higher levels of selfreported competency $(\bar{X}=11.66 \dot{6}, \sigma \approx 2.036)$ than those without $(\bar{X}=10.8, \sigma \approx 1.528)$. This has interesting implications for virtual reality systems. Our observations show a clear difference in presence to previous work, which largely ignored visuo-haptic feedback in a multisensory setting [26], [25], [28]. Correlation analyses did however, unearth some significant relationships between presence subscales across the two groups.

Table III

MANN-WHITNEY $U$ TEST RESULTS FOR PRESENCE SCORES ACROSS THE TWO GROUPS, WHERE $U$ IS THE $U$ TEST STATISTIC, $Z$ IS THE Z-SCORE AND $\alpha$ IS THE CONFIDENCE INTERVAL. IN ALL CASES, $N_{\text {INCONGRUent }}=25, N_{\text {CONGRUent }}=24, \alpha=.05$

\begin{tabular}{l|l|l|l|l|} 
Subscale & $U$ & $Z$ & $p$ & $p<\alpha$ \\
\hline \hline RL & 300.0 & 0.0 & 1.0 & No \\
PtA & 257.0 & -.865 & .387 & No \\
QoI & 289.5 & -.211 & .833 & No \\
PtE & 287.5 & -.252 & .801 & No \\
SEOP & 212.5 & -1.773 & .076 & No \\
Sounds & 276.0 & -.484 & .629 & No \\
Haptic & 286.5 & -.276 & .783 & No \\
$\Sigma$ & 294.0 & -.120 & .904 & No
\end{tabular}

\section{A. Correlating $P Q \&$ Human Factors}

A correlation analysis between presence subscales and pre-study questionnaire data was conducted, finding four significant relationships in total. The details of these cor- relations can be found in Table V. This specifically correlated the human factors listed in Table I (discarding completed lap time) and the presence subscale values for participants, found in Table IV. In our results, a positive relationship between previous VR experience and the Selfevaluation of Performance (SEOP) subscale can be observed, $r_{s}=.394, p<.05, N=49$, which is an expected result. However, previous racing game experience didn't yield any significant correlations, demonstrating the importance of previous VR exposure on task performance.

Table IV

A FULL LIST OF THE PRESENCE SUBSCALES RECORDED IN THE PQ QUESTIONNAIRE BY WITMER \& SINGER [29]

\begin{tabular}{l|l} 
Subscale name & Short name \\
\hline \hline Realism & RL \\
Possibility to Act & PtA \\
Quality of Interface & QoI \\
Possibility to Examine & PtE \\
Self-evaluation of Performance & SEOP \\
Auditory elements & Sounds \\
Haptic elements & Haptic \\
Sum of subscales & $\Sigma$
\end{tabular}

Table $\mathrm{V}$

A LIST OF SIGNIFICANT RESULTS FOUND THROUGH A CORRELATION ANALYSIS OF PQ SUBSCALES AND HUMAN FACTORS, WHERE $r_{s}$ IS THE CORRELATION COEFFICIENT OF THE TEST. IN ALL CASES, $N=49$.

\begin{tabular}{l|l|l|l} 
Variable 1 & Variable 2 & $r_{s}$ & $p$ \\
\hline \hline SEOP & Age group & -.328 & .021 \\
SEOP & Prior VR exp. & .394 & .005 \\
SEOP & Weekly hours driving & -.485 & .001 \\
PtA & Weekly hours driving & -.368 & .009
\end{tabular}

Another human factor correlated to PQ subscales concerned exposure to vehicle operation, namely the number of hours per week a participant spent driving. It was found that participant driving experience was negatively correlated to the SEOP $\left(r_{s}=-.485, p<.05, N=49\right)$ and Possibility to Act (PtA) presence subscales $\left(r_{s}=-.368, p<.05, N=\right.$ 49) across the two groups. Our findings suggest participants more experienced with vehicle control were less confident in their ability to carry out tasks, and had more trouble interacting with the VE. This is perhaps due to differences between simulated and real driving scenarios, with which an experienced driver would expect sensory cues closer to those of the real world. Another significant correlation was found between the age group of the participant, and the SEOP subscale. In particular, a negative correlation was found between these two variables, $r_{s}=-.328, p<.05, N=49$. This indicates that younger participants were more confident in their ability to carry out the driving task, whereas older participants were less confident.

\section{B. Correlating $P Q$ and Performance}

Another interesting set of variables to examine are measures of performance, and how they are related to subjective 
reports of presence. In particular, an analysis was conducted to examine correlations between measured presence subscales and participant lap times, similar to the previous correlation analyses. A summary of significant results can be found in Table VI, for both the first and second lap times. The first noteworthy result concerns negative relationships between presence subscales and the first lap time of participants. More specifically, the Realism (RL), Possibility to Act (PtA), Possibility to Examine (PtE) and Haptic subscales were all negatively correlated with the first lap time of the session. Similar correlations can be found for the second lap times, with negative relationships between second lap time and the Realism (RL), Possibility to Act (PtA) and Selfevaluation of Performance (SEOP) subscales. It is interesting that significant effects exist but across different subscales for the second lap. For example, SEOP scores were negatively related to lap-time performance in the second lap, but not the first. Regardless, our results indicate that presence affected driver performance more in the second lap, than the first. However, further investigation would be required to ascertain if this is the case beyond the scope of these experiments.

Table VI

A TABLE SHOWING ONLY THE SIGNIFICANT CORRELATIONS BETWEEN PQ SUBSCALES AND LAP TIMES, WHERE $r_{s}$ IS THE CORRELATION COEFFICIENT. INSIGNIFICANT CORRELATIONS ARE OMITTED FOR BREVITY. IN ALL CASES, $N=49$.

\begin{tabular}{l|l|l|l} 
Variable 1 & Variable 2 & $r_{s}$ & $p$ \\
\hline \hline First lap time & RL & -.352 & .013 \\
First lap time & PtA & -.403 & .004 \\
First lap time & PtE & -.362 & .011 \\
First lap time & Haptic & -.324 & .023 \\
First lap time & PQ $\Sigma$ & -.416 & .003 \\
First lap time & Second lap time & -.485 & .001 \\
Second lap time & RL & -.369 & .009 \\
Second lap time & PtA & -.406 & .004 \\
Second lap time & SEOP & -.323 & .024 \\
Second lap time & PQ $\Sigma$ & -.383 & .007
\end{tabular}

\section{Conclusions}

Despite the different visuo-haptic conditions the two groups were exposed to, there was no statistically significant result in presence levels between the two groups. This was observed across all of the recorded PQ subscales, however, the Self-evaluation of Performance (SEOP) subscale was the closest to significance in all cases. This is counter to previous work investigating visuo-haptic congruency, which notices a deterioration in presence levels with incongruent feedback [26], [27]. For example, Kuschel et al. [26] found a notable decrease in user presence as the perceived conflict of visuo-haptic information increased. Instead, our results show that regardless of whether visuo-haptic feedback is conflicting, immersion is not affected in the presence of a synchronous vestibular stimulus. The introduction of another modality in sensory integration may explain this effect, however, ascertaining whether this is the case is the subject for further research. A similar study by Kaas et al. [22] noted no positive effect of visual input on performance, which is also similar to our results. Our findings may have particular utility in virtual reality applications, especially those concerned with training and therapy. Furthermore, our results highlight the need for further research and understanding of this area, particularly visuo-haptic congruency in a multisensory environment.

Correlation analyses also showed some significant relationships in the recorded data. It was found that the level of previous experience using VR systems was positively correlated with higher self-reported evaluations of performance. Similarly, the degree of participant's driving experience was found to have a negative relationship with evaluations of performance. In particular, participants experienced in driving had lower estimates of performance in the simulated driving task, and vice versa. Previous work has shown that prior real-world expertise in simulated environments can negatively affect measures of presence and training effectiveness, for example, in flight simulator training tasks [31]. Our finding may be an extension of this principle. By the same token, measures of presence and lap time were found to be negative correlated, for both laps completed. Specifically, lower presence subscale values resulted in overall poorer performance across all laps completed. It is worth noting that some previous studies have found similar relationships between recorded presence levels and task performance [32], [33].

\section{A. Future Work}

One area we wish to consider in the future is to consider the same experiment with a larger sample size. Our sample size was relatively small, and it would be interesting to consider if the same effect is observed with an extended range of participants. Another area we wish to consider in future work is visually matching our experimental setup to the virtual input modalities. Whilst the gear changing modality visually matched the virtual one in its motion, its position was not identical. However, we plan to address this in future work as it would be a separately interesting research topic. Furthermore, research is required to explore other areas in which visuo-haptic congruency may have an impact. For example, it would be interesting to see if there are any notable differences in sickness levels or mental workload between the two groups. Furthermore, investigating the effect on player performance with more granularity, specifically focusing on in-game telemetry data such as acceleration rate, could yield interesting results. Whilst we noted that performance difference was insignificant, this does not account for driver behaviour which may have been impacted. It would also be interesting to consider the same experiment outside of the genre of a virtual racing game, especially in tasks which require considerably more dexterity. 


\section{ACKNOWLEDGEMENTS}

The authors would like to thank Grace Corn for agreeing to model the motion simulator and experimental setup in use, as well as the Student-Life team at the University of Lincoln for providing filming and photography support.

\section{REFERENCES}

[1] Alberto Gallace, Mary K Ngo, John Sulaitis, and Charles Spence. Multisensory presence in virtual reality: possibilities \& limitations. In Multiple sensorial media advances and applications: New developments in MulSeMedia, pages 1-38. IGI Global, 2012.

[2] Joseph J LaViola Jr. A discussion of cybersickness in virtual environments. ACM SIGCHI Bulletin, 32(1):47-56, 2000.

[3] Morton L Heilig. Sensorama simulator (1962). URL: http: //www.freepatentsonline.com/3050870.html, 2018.

[4] Peiji Wang, Adib A Becker, I Arthur Jones, AT Glover, SD Benford, CM Greenhalgh, and M Vloeberghs. A virtual reality surgery simulation of cutting and retraction in neurosurgery with force-feedback. Computer methods and programs in biomedicine, 84(1):11-18, 2006.

[5] Mark B Powers and Paul MG Emmelkamp. Virtual reality exposure therapy for anxiety disorders: A meta-analysis. Journal of anxiety disorders, 22(3):561-569, 2008.

[6] Nigel W John, Serban R Pop, Thomas W Day, Panagiotis D Ritsos, and Christopher J Headleand. The implementation and validation of a virtual environment for training powered wheelchair manoeuvres. IEEE transactions on visualization and computer graphics, 24(5):1867-1878, 2017.

[7] Leif P Berg and Judy M Vance. Industry use of virtual reality in product design and manufacturing: a survey. Virtual reality, 21(1):1-17, 2017.

[8] Bernhard E Riecke, Jörg Schulte-Pelkum, Marios N Avraamides, Markus Von Der Heyde, and Heinrich $\mathrm{H}$ Bülthoff. Cognitive factors can influence self-motion perception (vection) in virtual reality. ACM Transactions on Applied Perception (TAP), 3(3):194-216, 2006.

[9] Jake Harrington, Benjamin Williams, and Christopher Headleand. A Somatic Approach to Combating Cybersickness Utilising Airflow Feedback. In Computer Graphics and Visual Computing (CGVC). The Eurographics Association, 2019.

[10] Alessandra Gorini, José Luis Mosso, Dejanira Mosso, Erika Pineda, Norma Leticia Ruíz, Miriam Ramíez, José Luis Morales, and Giuseppe Riva. Emotional response to virtual reality exposure across different cultures: the role of the attribution process. Cyberpsychology \& behavior, 12(6):699$705,2009$.

[11] Jeffrey CF Ho. Real-world and virtual-world practices for virtual reality games: Effects on spatial perception and game performance. Multimodal Technologies and Interaction, 4(1):1, 2020.
[12] Gabriel Robles-De-La-Torre. The importance of the sense of touch in virtual and real environments. IEEE Multimedia, 13(3):24-30, 2006.

[13] Grigore C Burdea. Haptic feedback for virtual reality. In Virtual reality and prototyping workshop, volume 2, pages 17-29. Citeseer, 1999.

[14] Alejandro Jarillo Silva, Omar A Domínguez Ramirez, Vicente Parra Vega, and Jesus P Ordaz Oliver. Phantom omni haptic device: Kinematic and manipulability. In 2009 Electronics, Robotics and Automotive Mechanics Conference (CERMA), pages 193-198. IEEE, 2009.

[15] Shaoyu Cai, Pingchuan Ke, Shanshan Jiang, Takuji Narumi, and Kening Zhu. Demonstration of thermairglove: A pneumatic glove for material perception in virtual reality through thermal and force feedback. In SIGGRAPH Asia 2019 Emerging Technologies, pages 11-12. 2019.

[16] Olivier AJ Van der Meijden and Marlies P Schijven. The value of haptic feedback in conventional and robot-assisted minimal invasive surgery and virtual reality training: a current review. Surgical endoscopy, 23(6):1180-1190, 2009.

[17] Julian Kreimeier, Sebastian Hammer, Daniel Friedmann, Pascal Karg, Clemens Bühner, Lukas Bankel, and Timo Götzelmann. Evaluation of different types of haptic feedback influencing the task-based presence and performance in virtual reality. In Proceedings of the 12th ACM International Conference on Pervasive Technologies Related to Assistive Environments, pages 289-298, 2019.

[18] Tanja Kassuba, Corinna Klinge, Cordula Hölig, Brigitte Röder, and Hartwig R Siebner. Vision holds a greater share in visuo-haptic object recognition than touch. Neuroimage, 65:59-68, 2013

[19] Marc O Ernst and Martin S Banks. Humans integrate visual and haptic information in a statistically optimal fashion. Nature, 415(6870):429-433, 2002.

[20] James M Hillis, Marc O Ernst, Martin S Banks, and Michael S Landy. Combining sensory information: mandatory fusion within, but not between, senses. Science, 298(5598):16271630, 2002.

[21] Claudia Lunghi and David Alais. Congruent tactile stimulation reduces the strength of visual suppression during binocular rivalry. Scientific Reports, 5(1):1-9, 2015.

[22] Amanda L Kaas, Hanneke I van Mier, Johan Lataster, Mirella Fingal, and Alexander T Sack. The effect of visuo-haptic congruency on haptic spatial matching. Experimental Brain Research, 183(1):75-85, 2007.

[23] M. Di Luca and A. Mahnan. Perceptual limits of visualhaptic simultaneity in virtual reality interactions. In IEEE World Haptics Conference (WHC), pages 67-72, 2019.

[24] Frank Biocca, Jin Kim, and Yung Choi. Visual touch in virtual environments: An exploratory study of presence, multimodal interfaces, and cross-modal sensory illusions. Presence: Teleoperators \& Virtual Environments, 10(3):247-265, 2001. 
[25] Ali Sengül, Michiel van Elk, Olaf Blanke, and Hannes Bleuler. Congruent visuo-tactile feedback facilitates the extension of peripersonal space. In International Conference on Human Haptic Sensing and Touch Enabled Computer Applications, pages 673-684. Springer, 2018.

[26] Martin Kuschel, Franziska Freyberger, Martin Buss, and Berthold Färber. A presence measure for virtual reality and telepresence based on multimodal conflicts. In Proceedings of PRESENCE 2007: The 10th Annual International Workshop on Presence. Citeseer, 2007.

[27] Maria V Sanchez-Vives and Mel Slater. From presence to consciousness through virtual reality. Nature Reviews Neuroscience, 6(4):332-339, 2005.

[28] Samuel Couth, Daniel Poole, Emma Gowen, Rebecca A Champion, Paul A Warren, and Ellen Poliakoff. The effect of ageing on optimal integration of conflicting and nonconflicting visual-haptic stimuli. Multisensory research, 32(8):771-796, 2019.

[29] Bob G Witmer and Michael J Singer. Measuring presence in virtual environments: A presence questionnaire. Presence, 7(3):225-240, 1998.

[30] Geneviève Robillard, Stéphane Bouchard, Thomas Fournier, and Patrice Renaud. Anxiety and presence during vr immersion: A comparative study of the reactions of phobic and non-phobic participants in therapeutic virtual environments derived from computer games. CyberPsychology \& Behavior, 6(5):467-476, 2003.

[31] Joost CF De Winter, Dimitra Dodou, and Max Mulder. Training effectiveness of whole body flight simulator motion: A comprehensive meta-analysis. The International Journal of Aviation Psychology, 22(2):164-183, 2012.

[32] Jonathan A Stevens, J Peter Kincaid, et al. The relationship between presence and performance in virtual simulation training. Open Journal of Modelling and Simulation, 3(02):41, 2015.

[33] Christine Youngblut and Odette Huie. The relationship between presence and performance in virtual environments: Results of a verts study. In IEEE Virtual Reality, 2003. Proceedings., pages 277-278. IEEE, 2003. 